\title{
SIGNIFICADOS DE LAS PRÁCTICAS SEXUALES ENTRE AYMARA CHILENOS. APORTES PARA REFLEXIONAR SOBRE LOS CUIDADOS TRANSCULTURALES
}

Meanings of Practice about Sexuality between Chilean Aymara People. Contributions to Ponder about Transcultural Care

\author{
ANA MARÍA CARRASCO*, VIVIAN GAVILÁN**, PATRICIA VIGUERAS*** \& MARÍA BELÉN \\ VÁSQUEZ $Z^{\star \star \star \star}$
}

Fecha de recepción: 08 de marzo de 2021 - Fecha de aprobación: 27 de mayo de 2021

\section{Resumen}

Desde la mirada transcultural de los cuidados, el conocimiento de los significados de las prácticas acerca de la sexualidad de los pueblos originarios de América Latina es relevante para diseñar estrategias de cuidado y avanzar en la prevención y promoción de la salud sexual y reproductiva con pertinencia cultural. Siguiendo los estudios de la antropología de género, de la sexualidad y de los cuidados, este artículo presenta resultados de una investigación cualitativa, cuyos sujetos de estudio fueron mujeres y hombres adscritos al Pueblo Aymara del norte chileno. Se identifican aspectos clave de las representaciones del cuerpo, sexualidad, gestación, reproducción y del control de la natalidad que particularizan al conjunto social aymara. Estos antecedentes son de utilidad para reflexionar en torno a las consecuencias negativas que pueden desplegar las políticas sociales en salud sexual y reproductiva al centrarse en un sujeto universal y a las dificultades que pueden entorpecer los cuidados enfermeros cuando se invisibilizan las diferencias de los significados que asignan los sujetos a su sexualidad.

Palabras clave: enfermería transcultural; sexualidad; género; población indígena; aymara.

\begin{abstract}
From a transcultural view of the care, knowledge of the meanings of practices about sexuality of the indigenous groups of Latin America is important to design strategies of care and for advancing in prevention and promotion of sexual and reproductive health with cultural pertinence. Following the studies of anthropology of gender, sexuality and care, this article presents the results from a qualitative study whose study subjects were women and men belonging to the Aymara population of northern Chile. Key aspects of the representations of the body, sexuality, pregnancy, reproduction and birth control that particularize to the Aymara social group are identified. This background is useful to debate about the negative consequences that can deploy the social policies in sexual and reproductive health by focusing on a universal subject and on the difficulties that can hinder the nursing care when the differences in the meanings of their sexuality assigned by a person become invisible.
\end{abstract}

Keywords: transcultural nursing; sexuality; gender; indigenous population; Aymara.

* Dra. en Antropología. Universidad de Tarapacá, Departamento de Antropología, Facultad de Ciencias Sociales y Jurídicas, Arica, Chile. Artículo enmarcado en el proyecto: "Adolescencia y sexualidad. Construcción sociocultural de la homosexualidad y el homoerotismo en adolescentes varones, aymara y no aymara del norte de Chile", FONDECYT Regular № 1190822. Correo-e: anycarrasco@gmail.com

** Magíster en Antropología. Universidad de Tarapacá, Departamento de Antropología, Facultad de Ciencias Sociales y Jurídicas, Arica, Chile. Correo-e: viviangav@yahoo.com

*** Dra. (c) en Antropología médica y Salud Global. Facultad de Ciencias de la Salud, Carrera de Enfermería, Universidad Arturo Prat, Escuela de Enfermería. Iquique, Chile. Correo-e: patriciaviguerascherres@gmail.com

**** Doctora (c) en Antropología. Universidad de Tarapacá, Departamento de Antropología, Facultad de Ciencia Sociales y Jurídicas, Iquique, Chile. Correo-e: belenvasquezs@gmail.com 


\section{Introducción}

La enfermería transcultural ha planteado la necesidad de incorporar la diversidad cultural como un componente central en el análisis de los procesos de salud-enfermedad-atención y, en consecuencia, ha sugerido que los cuidados deben considerar a la persona social. Esto implica concebir al individuo dentro de su entorno sociocultural, socioeconómico y sociopolítico (Aguilar, Carrasco, García, Saldívar \& Ostiguín, 2007; Manrique, Reyes, Delgado, Jiménez \& Leininger, 2005; Marriner-Tomey \& Raile Alligood, 1999; Rohrbach-Viadas, 1998).

En el campo de la salud, la sexualidad ocupa un lugar relevante. No obstante, su estudio ha sido escasamente abordado desde la perspectiva de los cuidados culturales. En Chile, como en otros países latinoamericanos, la investigación sobre sexualidad toma fuerza alrededor de dos décadas atrás, siendo en un primer momento mayoritariamente de corte epidemiológico, para detectar prácticas de riesgo en la transmisión de ciertas enfermedades "sexuales" o estudiar los comportamientos sexuales de cierto estrato de población, principalmente joven, y su vinculación con el uso de anticonceptivos. Luego se incorporan aspectos sobre derechos sexuales y reproductivos, tema vinculado con reformas de salud y educacional (Pérez \& Dides, 2005; Shiappacase, 2003; Palma, 1996, 2002; Palma \& Canales, 1999; Gysling, 1995; entre otros).

Por otro lado, una particularidad de gran parte de las investigaciones sobre sexualidad humana es que se han centrado en enfoques biomédicos, los cuales sostienen que son básicamente las características biológicas de cada sexo las que la determinan. Visto de esta forma, el cuerpo humano tendría un funciona- miento sexual universal que estaría determinado por la fisiología y por impulsos naturales. Encontramos, en concordancia, una tendencia esencialista en la investigación biomédica en la que el énfasis ha estado dado en las determinaciones biológicas del sexo "de modo que las eventuales diversidades de comportamientos o actitudes se evalúan en relación con una normalidad que no es asumida en su relatividad social, sino como norma ideal" (Lamadrid \& Muñoz, 1996, p. 128).

Ahora, si bien desde las ciencias sociales la sexualidad y la cultura han sido un tema de interés, hay que precisar que es solo en las últimas décadas que los argumentos antiesencialistas aumentan y se acepta que la sexualidad no es "natural", sino que ha sido y es construida (Lamas, 2000). De esta manera se comienza a ampliar su conocimiento, lo que ha permitido la generación de reflexiones teóricas, metodológicas y éticas que la legitiman como su objeto de estudio. Estos avances investigativos muestran la sexualidad humana como un fenómeno complejo que debe ser entendido desde la subjetividad de los actores sociales $y$ de las relaciones sociales e instituciones involucradas en su configuración, de lo cual es evidencia la existencia de una diversidad de prácticas y de significados sobre la sexualidad en distintos grupos humanos (Nieto, 2003; Weeks, 1998; Lamadrid \& Muñoz, 1996; Bozon \& Leridon, 1993). El énfasis se pone, entonces, en la necesidad de conocer no solamente las prácticas sexuales, sino las significaciones que hay tras esos comportamientos, las relaciones sociales, las instituciones que le dan sentido, el vínculo entre los deseos y los comportamientos sexuales y la subjetividad de las personas. La mirada se abre, permitiendo la posibilidad de dar cuenta de discursos, acciones institucio- 
nales, normas, valores y transgresiones que moldean lo que la gente habla y lo que calla.

De esta manera, estos nuevos aportes de la antropología de la sexualidad y de la reproducción advierten que lejos de instalarse únicamente en el orden biológico, la sexualidad está mediada por las concepciones culturales y condiciones sociales que las sociedades construyen a través de su historia y que permiten la elaboración de diversas formas de dar satisfacción a las necesidades sexuales y reproductivas (Blázquez, 2005; Szasz, 1998; Weeks, 1998; Lamadrid \& Muñoz, 1996; Bozon \& Leridon, 1993).

Lamentablemente, la perspectiva social mencionada se ha vinculado débilmente con la salud. De esta manera, muchas veces en este campo se ha invisibilizado el hecho de que la sexualidad, al constituir una dimensión de la realidad sociocultural y sociohistórica, determina dinámicas específicas tanto institucionales como de los sujetos concretos. Ello conlleva que los significados que los grupos asignan a la sexualidad difieren entre sí y se transforman a través del tiempo. La regulación de la sexualidad humana se cristaliza en instituciones y se manifiestan en contextos de relaciones de poder (Foucault, 2008).

Por otra parte, siguiendo nuestra postura, reflexión y contextualización, y en relación con los sujetos de estudio, debemos recordar que los pueblos originarios en Chile forman parte de este Estado-nación y, por lo tanto, son destinatarios de las políticas públicas en salud sexual y reproductiva. Sin embargo, dichas políticas públicas no consideran las especificidades culturales al momento de regular y establecer la salud sexual. Han sido poco visibles en los diagnósticos, políticas y programas que, sobre el tema, a nivel nacional, se han realizado.
Sabemos que Chile no es homogéneo social y culturalmente, en su interior conviven varios grupos étnicos, entre los cuales se halla el Pueblo Aymara. Si asumimos que ellos han formado parte de los procesos sociohistóricos chilenos y que además no son ajenos al panorama de profundas transformaciones que se viven y que generan un contexto social nacional heterogéneo cambiante, creemos resulta válido preguntarse y conocer las características particulares en las que se desenvuelve su sexualidad, así como también por los contextos institucionales, morales y éticos en que organizan las prácticas sexuales.

Esto nos permitirá abordar la necesidad que creemos hoy existe de mayor información sobre la construcción de los significados sexuales y sus prácticas en un país multicultural como el nuestro. Por otra parte, vale también mencionar que son escasos los estudios acerca de los conceptos sobre los cuidados culturales relacionados con la sexualidad de los miembros de las comunidades indígenas en general y del norte de Chile en particular (Vásquez \& Carrasco, 2017; Carrasco \& Gavilán, 2014a; Dides \& Moscoso, 2007; Pérez \& Dides, 2005; Alarcón, Vidal \& Neira, 2003; CEDEMU, 2001). En este sentido y en el marco de una investigación que versó sobre la sexualidad en personas adscritas étnicamente en las regiones de Tarapacá y Arica y Parinacota, nos interesó rescatar aquellos resultados que contribuyen a la generación de nuevas prácticas de cuidados enfermeros pertinentes para el Pueblo Aymara.

A este respecto, queremos dejar claro que la población de origen aymara no es solo una minoría lingüística y cultural, sino que constituye un grupo étnico en la medida en que tanto ella como la sociedad nacional han insistido en 
la diferenciación por varios siglos (Gundermann, Vergara, \& Díaz, 2011; Echeverría \& Guzmán, 2009; González \& Gavilán, 1990; Chipana, 1986). Los aymara asumen una descendencia de antepasados comunes, convicción basada en argumentos que atestiguan un pasado conjunto: rasgos somáticos, lengua, cultura y tradiciones compartidas; religión, mitos y memorias; usos y costumbres (D'Andrea, 2000). Su historia actual es mitológica e incluye algunos conceptos de continuidad generacionales. Esto les ha permitido reivindicar cierta autonomía frente al Estado-nación y a los segmentos regionales de los grupos cultural y políticamente dominantes (Gavilán, 2005). A lo largo de varios siglos han mantenido relaciones de acomodamiento y de tensión, y elaborado fronteras psicológicas y no territoriales. Estas fronteras son mantenidas por adscripción, así como por recursos externos, entre los que destaca la permanente diferenciación que hace la sociedad regional y nacional (González, 1998; De Vos \& Romanucci-Rossi, 1982).

Producto de los procesos históricos hoy existe una gran heterogeneidad en la población aymara, por lo que se pueden señalar varios subgrupos. En una gradación básica, encontraríamos dos extremos: la población que mantiene el aymara como lengua materna, aún muy vinculada al sector rural campesino y que sigue las prácticas rituales tradicionales (la "costumbre") (Gundermann, González \& Vergara, 2007); y en el otro, aquellos pobladores urbanos que no hablan ni entienden su lengua vernácula, pero que se adscriben a tradiciones en las que incluyen la historia andina reconstruida (Carrasco \& González, 2014). Existen asimismo complejos procesos de fuga del grupo étnico, por lo que también es posible observar una gran cantidad de población que se ha desvinculado totalmente de su pasado (Carrasco \& Gavilán,
2012, 2014b; Carrasco, 2011, 2007; González \& Gavilán, 1990).

Entendida como filtro, la tradición que comparten ofrece herramientas para la interpretación y las elaboraciones sucesivas de las relaciones sociales, la religiosidad y la sexualidad (Gavilán \& Carrasco, 2009). En este sentido, lo que observamos en la mitología y la religiosidad aymara en la actualidad ofrece un contexto moral y una ideología que orienta las prácticas sexuales de los individuos adscritos a esta etnia (Carrasco \& Gavilán, 2005, 2014a, 2014b). Así visto, deberíamos asumir que la noción de sexualidad entre los aymara contemporáneos posee características particulares y distintas de la sociedad mayor chileno-mestiza. ¿Cuáles son estas características particulares? ¿Cuál es la noción de sexualidad entre los aymara contemporáneos? Estas y otras interrogantes se nos presentan en un estudio como este y varias son las vías de entrada para responderlas. Nosotras vimos como una posibilidad indagar en los paradigmas éticos y morales para identificar las representaciones que construyen de la sexualidad humana para, de esta forma, conocer los significados asignados que se materializan en sus comportamientos sexuales. Esta opción metodológica es la que presentamos en este artículo.

\section{Material y método}

Los datos utilizados corresponden a una investigación sobre sexualidad entre los aymara del norte chileno realizada entre 2012 y 2014 . Metodológicamente, se planteó un estudio cualitativo que buscó conocer los significados de sus prácticas sexuales y reproductivas. 
La información se obtuvo mediante relatos de vida y entrevistas en profundidad y semiestructuradas. Para las entrevistas se diseñaron dos instrumentos: uno para abarcar el ámbito de la sexualidad propiamente tal y otro para rescatar conocimientos sobre el cuerpo y la concepción aymara del mismo. La entrevista sobre sexualidad abarcó 40 personas, todas aymaras, seleccionadas con tres criterios diferenciadores: sexo, edad y adscripción religiosa. Veinte eran hombres y 20 mujeres, de tres tramos de edad, entre los 15 y 65 y más años. Sus adscripciones religiosas fueron: católicos, evangélicos y sin opción religiosa. La entrevista sobre cuerpo y concepción fue aplicada a 10 individuos: 5 hombres y 5 mujeres adscritos al Pueblo Aymara, practicantes de la medicina ancestral. Finalmente, los relatos de vida se aplicaron a 15 personas, aymara, hombres y mujeres de tres categorías de edad: jóvenes, adultos y adultos mayores.

Respecto a la condición de aymara de los entrevistados(as) se utilizó como criterio principal el de la autoadscripción, considerándose también la identificación de sus pares, lugar de origen y apellidos. Para la adscripción religiosa se privilegió la socialización del individuo en ambientes católicos, evangélicos o autodefinidos como sin opción religiosa. Por otra parte, la variable edad remite tanto al ciclo de vida del sujeto -es decir, a etapas en la vida de cada entrevistado(a) que define posiciones distintas tanto en la familia como en la sociedad en general- como a generaciones y épocas culturales diferenciadas en la sociedad en su conjunto (Carrasco, 1998). Así se distinguieron tres categorías de sujetos: jóvenes (15-25 años), adultos (26-45 años) y adultos mayores (46-65 y más años).

Sobre el tratamiento de la información recolectada, una vez realizadas las entrevistas se transcribieron textualmente, se revisaron las transcripciones, se ordenó la información y se sistematizó según las dimensiones consideradas (socialización familiar en sexualidad, relaciones sexuales y cuerpo) y categorías definidas para cada una de ellas.

\section{Resultados}

Los datos empíricos recogidos indican que las representaciones de la "naturaleza" del cuerpo humano y de su reproducción varían según la generación, el grado de intervención que haya tenido la familia campesina en la socialización del sujeto y la apropiación que los sujetos tienen de los conocimientos sobre sexualidad, reproducción y afectividad impartidos por el sistema escolar.

La forma de representar los cuerpos y las diferencias sexuales de los aymara configuran saberes inscritos en los dominios "biológico", "fisiológico" y "espiritual". En los casos de las mujeres y los hombres de mayor edad y menor escolaridad, estos tienden a no separar los dominios religiosos y biológicos, por lo que la forma de elaborar la relación entre cuerpos sexuados y representaciones simbólicas de los mismos ofrece una constelación de conocimientos que fundamentan los significados de las prácticas sobre sexualidad y reproducción de hombres y mujeres. Esta tendencia va disminuyendo de acuerdo con la intervención de la escuela en la socialización de las personas. Ello indica que la escuela incide en la secularización de los conjuntos sociales, lo cual implica que las representaciones basadas en la biología, como una de las ciencias hegemónicas, van en aumento y generan cambios en las normas y las reglas sobre la socialización de las personas. De esta manera, se observan 
cambios entre las generaciones. Un ejemplo de ello se presenta en la estética del cuerpo, ya que las y los jóvenes tienden a asemejarse a los no indígenas en su vestimenta, peinados y exposición de sus partes.

En la población con menor escolaridad y mayor experiencia campesina, independientemente de su edad y residencia urbana o rural, está presente la idea de que los cuerpos humanos y de los animales sexuados sirven de base a la representación del paisaje. Así, los cerros masculinos, nominados como Mallku y en los rituales con nombres propios, poseen características de hombres (cabeza, piernas, brazos $y$, en general, se perciben con el fenotipo de la población prehispánica). Los cerros hembras, llamados T'alla y en los rituales con nombres propios, se caracterizan por su aspecto morfológico femenino asociado al fenotipo de la mujer indígena. Muchas veces se les ve por la noche vestidos a la usanza tradicional ${ }^{1}$ (Gavilán \& Carrasco, 2009).

El funcionamiento del organismo animal se piensa similar al humano. De la observación de las diferencias y semejanzas anatómicas y fisiológicas se desprenden nociones abstractas que orientan los modelos de género y su jerarquización. Se observan categorías de las diferencias sexuales basadas en oposiciones binarias; así, por ejemplo, en relación con la gestación, lo femenino está asociado a sangre menstrual (principio de la vida) y la grasa a lo masculino (principio de la vida). La sangre roja se une con la sangre blanca del hombre (semen) produciendo la fecundación. El feto femenino se gesta en menor tiempo que el masculino, puesto que la naturaleza del cuerpo de la mujer es blando, liviano y de barro; en tanto que el hombre posee huesos duros, pesados y vinculado a la piedra.
La gestación de las mujeres se inicia por las piernas y luego la matriz-corazón; en cambio, los hombres se desarrollan desde la cabeza, los brazos, la matriz, las entrañas. Las mujeres poseen una matriz grande, mientras que los hombres un útero pequeño, llamado normalmente padre (podría referirse a la próstata).

Los términos relacionados a la sexualidad según el género fueron los siguientes: la vagina que envuelve y ordeña, el pene que coagula. Lo femenino es continente y receptor, lo masculino es contenido y depositario: la capacidad sexual de la mujer es ilimitada, la del hombre limitada; lo femenino se asocia a movimientos suaves y lentos, en cambio lo masculino se relaciona con movimientos fuertes y rápidos.

Si bien estas ideas van siendo reemplazadas por los argumentos de la biología y de la medicina moderna, las representaciones de la sexualidad y de la reproducción de mujeres y hombres mantienen aspectos del saber tradicional aymara. Entre estos, destaca la persistencia de la creencia de que los cuerpos, al ser sexuados, necesitan satisfacer el deseo sexual, lo cual es visible en los mitos, leyendas y argumentaciones de las personas. Estas ideas se manifiestan en los rituales, especialmente en la festividad de los carnavales, ya que se cree que las divinidades viven en pareja y practican el sexo y, a su semejanza, la humanidad, los animales, las plantas y el universo.

Entre los cambios observados, se ve que mujeres y hombres adultos se iniciaron sexualmente a más temprana edad. Las y los jóvenes residentes en la ciudad se inician sexualmente a mayor edad que las mujeres campesinas, por lo cual es probable que en el espacio urbano exista un mayor control de la sexualidad por 
parte de madres y padres. Aunque este aspecto no constituye un tema de conversación abierto, se transfiere información a través de chistes, leyendas y fábulas.

Entre las familias campesinas del altiplano, el conocimiento empírico de la sexualidad del ganado y el hecho de compartir dormitorios con los adultos, constituyen una fuente importante para la experiencia personal y colectiva de su propia sexualidad. Entre los más jóvenes y con residencia urbana, los grupos de pares, las revistas y los medios de comunicación aparecen con mayor incidencia como fuentes de información. Entre los hombres adultos, la concurrencia a prostíbulos en la ciudad parece ser una práctica en sus primeras experiencias.

La prevención del embarazo no constituye un problema importante para las personas aymara adultas. Los hijos e hijas nacidos vivos de parejas fuera del matrimonio son criados, por lo general, por las familias del padre, de acuerdo a arreglos consensuados siguiendo el patrón de descendencia patrilineal. Por otra parte, se debe considerar que en la sociedad aymara campesina la sexualidad adolescente fue extensamente permitida, aunque no reconocida verbalmente. Asimismo, existió la fase de prueba del matrimonio que consideraba la unión de la pareja por un periodo de dos años aproximadamente antes de consolidar el matrimonio civil o eclesiástico. El uso de anticonceptivos tuvo una gran resistencia entre las mujeres con residencia rural, especialmente entre las parejas y/o maridos. Esta situación ha ido variando hacia un mayor uso; no obstante, sigue siendo más restrictiva que entre los no indígenas.

Los datos indican, también, que las relaciones sexuales forman parte de un proceso que culmina con la unión conyugal, que es socialmente valorada. Podríamos decir que la sociedad aymara rechaza hoy el deseo sexual femenino no orientado a la conyugalidad y a la procreación. Sin embargo, no se niega la sexualidad femenina, sobre todo durante el período menstrual.

Un aspecto interesante de los resultados y que resaltan las mujeres aymara es la ausencia de romanticismo en sus primeras experiencias sexuales. Entre las adultas y mayores se cree que tanto hombres como mujeres deberían tener igual interés en el sexo; esto porque existirían dos categorías centrales en las que se ubicarían todos los seres humanos: aquellos(as) que tienen sangre caliente y son más ardorosos(as) y los(as) de sangre fría, con menor inclinación por el sexo. Cuando ambos integrantes de una pareja tienen sangre fría, se espera que conformen una familia pequeña ya que no tendrían muchos(as) hijos(as) y viceversa. La edad también influiría en esta diferenciación, ya que las mujeres de más edad, ya pasando los cincuenta años, se irían enfriando y, por lo tanto, tendrían menos deseos sexuales que las jóvenes.

La sexualidad femenina sería distinta a la masculina en el sentido de que sexualmente al hombre se le considera más cercano a la naturaleza, al instinto, él toma la iniciativa y maneja la situación. La mujer debe jugar un papel pasivo de receptora, porque así está establecido social y culturalmente, desde pequeñas se les inculca una actitud sumisa frente a los hombres. No obstante, la creencia de que el sexo de los bebés se define por el orgasmo femenino o masculino, es decir, si la mujer termina antes que el hombre el bebé será de sexo femenino, afirmaría que el goce sexual está presente en ellas.

La mayoría de las entrevistadas adultas tiene una vida de pareja larga, con un compañero 
sexual único, pero con más de uno entre las adultas mayores. Los celos de los hombres son uno de los motivos de conflicto marital, actitudes que supongan "coqueteo" de la mujer o un grado de confianza mayor con otros hombres es mal visto. La definición de placer como el "goce de la pareja" implica a ambos sexos. La autocomplacencia sexual y el recurso de la masturbación no es bien vistos para ambos géneros y solo es aceptado para los hombres durante la adolescencia.

La sexualidad de mujeres y hombres no aymara está más regulada por la ideología cristiana que por la biología y la medicina. No obstante, esta ideología tiene menor influencia entre los aymara; los jóvenes son los únicos que tienden a suscribir de manera más acentuada los preceptos cristianos. Es decir, relacionan la sexualidad con la reproducción dentro del matrimonio dejando de lado el placer. La marcación de la maternidad como un valor trascendente, presente en la ideología cristiana y como componente central de la identidad de género, conduce a que el erotismo y la lascivia femenina se piensen como signos de pecado y prohibición. Entre los aymara, la incorporación de la sexualidad como componente de las relaciones entre las deidades tutelares lleva a que la maternidad no sea excluyente de la sexualidad de la mujer. Asimismo, la paternidad como autoridad inobjetable impuesta por el cristianismo es extensiva a la población aymara, aunque entre estos la maternidad tiende a compensar ese poder, especialmente en la unidad doméstica campesina.

\section{Discusión y comentario}

Los antecedentes expuestos muestran que los significados de las prácticas sexuales y reproductivas tienen una especificidad entre los aymara del norte de Chile. A pesar de los esfuerzos estatales por homogenizar culturalmente a la población a través de medidas universales de regulación de la fecundidad y de la sexualidad, existen formas de pensar la vida sexual entre las mujeres y los hombres aymara que se distancian del modelo hegemónico, que tiende a estandarizar el comportamiento sexual a partir de categorías occidentales modernas.

El desconocimiento de estas diferencias por parte de los equipos de salud hace que el respeto por las creencias, declaradas en las políticas públicas en salud sexual y reproductiva, no sea suficiente para aplicar cuidados con pertinencia cultural. La insistencia por enfrentar esta aproximación desde la prevención del embarazo, la regulación de la fecundidad y la prevención de las enfermedades de transmisión sexual olvida la multidimensionalidad del fenómeno de la sexualidad y tiende a considerarla como un factor de riesgo para la salud y no como parte de su promoción. En este sentido, el hecho de que los significados que los actores sociales asignan a su sexualidad depende de sus características socioculturales exige a los profesionales de enfermería problematizar la universalidad de los cuidados, pues aquellos que son considerados como normales pueden ser contraproducentes. Por lo tanto, el diseño de cuidados enfermeros con pertinencia cultural requiere reconocer la diferencia y asumirla como otra forma posible de comprender la sexualidad y no imponer aquella que una parte de la sociedad nacional considera la más adecuada. De aquí que la enfermería transcultural es una propuesta que puede contribuir a abrir caminos hacia un diálogo intercultural. 


\section{Notas}

${ }^{1}$ Mallku-Tálla significa, en sentido literal, señor y señora principal de la comunidad. Ambos representan lo masculino y lo femenino en la relación de pareja social y sexual. T'alla, como palabra vinculada a la mujer, contiene ideas relacionadas con su potencialidad sexual.

\section{Referencias bibliográficas}

Aguilar, O., Carrasco, M., García, M.A., Saldívar, A. \& Ostiguín, R. M. (2007). Madeleine Leininger: un análisis de sus fundamentos teóricos. Revista Enfermería Universitaria, 4(2), 26-30.

Alarcón, A.M., Vidal, A. \& Neira, J. (2003). Salud intercultural: elementos para la construcción de sus bases conceptuales. Revista Médica de Chile, 131, 1061-1065.

Blázquez, M. (2005). Aproximación a la antropología de la reproducción. Revista de Antropología Iberoamericana [online], 42. Recuperado de http://www.aibr.org/ antropologia/42jul/articulos/ jul0506.pdf.

Bozon, M. \& Leridon, H. (1993). Les constructions sociales de la sexualité. Population, 48(5), 1173-1195.

Carrasco, A.M. (1998). Constitución de género y ciclo vital entre los aymaras contemporáneos del norte de Chile. Chungara, Revista de Antropología Chilena, 30(1), 87-103.

(2007). Influencias de la ideología religiosa en las significaciones de la sexualidad en mujeres aymaras del norte de Chile. Cultura y Religión, 1(2).

(2011). Paradigmas éticos y morales en la construcción de la sexualidad de hombres y mujeres indígenas y no indígenas en el norte de Chile. Nuevas Tendencias en Antropología, 1, 108-126.

Carrasco, A. M. \& Gavilán, V. (2005). Sexualidad y género: La unidad de lo femenino y lo masculino como símbolos de reproducción y fertilidad entre los aymara del norte de Chile. En Rebolledo, L.; Tomic, P. \& Garduño, E. (Eds.), Imaginarios, identidades e historias: Miradas desde la antropología del género (pp. 169-186). Mexicali: Centro de Estudios Culturales, Universidad Autónoma de Baja California, Instituto de la Mujer para el Estado de Baja California.

(2012). Influencia de los programas escolares de enseñanza primaria en la socialización de mujeres y hombres aymara de la zona altiplánica del norte de Chile. Estudios Atacameños, 44, 73-88.

(2014a). Significados y prácticas de la sexualidad en tres generaciones de mujeres aymaras del norte de Chile. Revista Interciencia, 39(7), 468-475.

(2014b). Género y etnicidad: Ser hombre y ser mujer entre los aymaras del altiplano chileno. Diálogo Andino, 45, 69-180.

Carrasco, A. M. \& González, H. (2014). Movilidad poblacional y procesos de articulación rural-urbano entre los aymara del norte de Chile. Si Somos Americanos, Revista de Estudios Transfronterizos, 14(2), 217-231.

CEDEMU (2001). Sentando bases para el desarrollo de un modelo intercultural en salud sexual y reproductiva entre las mujeres aymará del norte de Chile: un estudio de epidemiología intercultural, balance del proceso. Arica.

Chipana, C. (1986). La identidad étnica de los aymarás en Arica. Chungara, Revista de Antropología Chilena, 16-17, 251-261.

D’Andrea, D. (2000). Las razones de la etnicidad entre globalización y eclipse de la política (traducido por Gilberto Jiménez). En Cerutti, F. \& D’Andrea, D. (Comps.), Identitá e conflitti. Milán: Franco Angeli.

De Vos, G. \& Romanucci-Rossi, L. (1982). Ethnic identity. Chicago: University of Chicago Press.

Dides, C. \& Moscoso, M. S. (2007). Investigaciones en salud sexual y reproductiva de pueblos indígenas en Chile y la ausencia de pertinencia étnica. Acta Bioethica, 13(2), 216-222.

Echeverría, R. \& Guzmán, P. (2009). Resignificación de la identidad aymara en el contextoeconómico actual de la Zona de Arica. (Tesis inédita de pregrado en antropología). Universidad Academia de Humanismo Cristiano, Santiago.

Foucault, M. (2008). Historia de la sexualidad: La voluntad de saber. México: Siglo XXI.

Gavilán, V. (2005). Identidades sociales a inicios del siglo XXI. Dialectología y Tradiciones Populares, 2, 77-102.

Gavilán, V. \& Carrasco, A. (2009). Festividades andinas y religiosidad en el norte chileno. Chungara, Revista de Antropología Chilena, 41, 101-112.

González, H. (1998). Apuntes sobre el tema de la identidad cultural en la Región de Tarapacá. Estudios Atacameños, 13, 27-45.

González, H. \& Gavilán, V. (1990). Cultura e identidad étnica entre los aymaras chilenos. Chungara, Revista de Antropología Chilena, 24-25, 145-158.

Gundermann, H., González, H. \& Vergara, J. (2007). Vigencia y desplazamiento de la lengua aymara en Chile. Estudios Filológicos, 42, $123-140$.

Gundermann, H., Vergara, J. \& Díaz, A. (2011). Historia moderna de una lengua originaria: El jaqi aru en Chile. Revista de Lingüística Teórica y Aplicada, 49(1), 69-108.

Gysling, J. (1995). La investigación social en salud reproductiva en Chile: Panorama al inicio de los noventa. Santiago: Universidad de Chile, PIEG, Facultad Latinoamericana de Ciencias Sociales-Chile.

Lamadrid, S. \& Muñoz, S. (1996). La investigación social en sexualidad en Chile, 1984-1994. Santiago: Programa Interdisciplinario de Estudios de Género, Universidad de Chile.

Lamas, M. (2000). Sexualidad y género: La voluntad de saber feminista. En Szasz, I. \& Lerner, S. (Eds.), Sexualidades en México: Algunas aproximaciones desde la perspectiva de las ciencias sociales (pp. 49-69). México: El Colegio de México. 
Manrique, M., Reyes, I., Delgado, H., Jiménez, B. \& Leininger, M. (2005). Cuidados culturales: Teoría de la diversidad y la universalidad. Recuperado de http://www.enfermeria21.com/listametas/Leininger_ monografia.doc

Marriner-Tomey, A. \& Raile Alligood, M. (1999). Modelos y teorías en enfermería. Madrid: Harcourt Brace.

Nieto, J. A. (Ed.) (2003). Antropología de la sexualidad y diversidad cultural. Madrid: Talasa.

Palma, I. (1996). Discursos sobre sexualidad y salud reproductiva en adultos jóvenes: Factores facilitadores e inhibitorios en la prevención de riesgos. Informe de Investigación, Santiago.

(2002). Salud y derechos sexuales y reproductivos de adolescentes y jóvenes en el contexto de la reforma de salud en Chile. En OPS/OMS, Género, equidad y reforma de la salud en Chile: Voces y propuestas desde la sociedad civil (pp. 1-11). Santiago: OPS/OMS.

Palma, I. \& Canales, M. (1999). Demandas y necesidades sobre sexualidad y afectividad de los/as adolescentes no insertos en el sistema educativo formal. Santiago: Servicio Nacional de la Mujer.

Pérez, M. S. \& Dides C. (2005). Salud, sexualidad y reproducción: Sistematización de investigaciones y experiencias en pueblos indígenas en Chile 1990-2004. Santiago: CORSAPS, UNFPA.
Rohrbach-Viadas, C. (1998). Introducción a la teoría de los cuidados culturales enfermeros de la diversidad y de la universalidad de Madeleine Leininger. Cultura de los Cuidados, 3, 1138-1728.

Shiappacase, V. (2003). Chile: Situación de la salud y los derechos sexuales y reproductivos. Santiago, Instituto Chileno de Medicina Reproductiva, Corporación de Salud y Políticas Sociales, SERNAM.

Szasz, I. (1998). Primeros acercamientos al estudio de las dimensiones sociales y culturales de la sexualidad en México. En Szasz, I. \& Lerner, S. (Eds.), Sexualidades en México: Algunas aproximaciones desde la perspectiva de las ciencias sociales (pp. 11-33). México: El Colegio de México.

Vásquez, M. B. \& Carrasco, A. M. (2017). Los estudios antropológicos de la sangre menstrual: Representaciones y prácticas de las mujeres aymaras del norte de Chile. Revista Chungara, Revista de Antropología Chilena, 49(1), 99-108.

Weeks, J. (1998). La construcción de las identidades genéricas y sexuales: La naturaleza problemática de las identidades. En Szasz, I. \& Lerne, S. (Eds.), Sexualidades en México: Algunas aproximaciones desde la perspectiva de las ciencias sociales (pp. 175-198). México: El Colegio de México. 